\title{
MODELLING OF PRESSURE PULSATION IN GAS COMPRESSOR STATION
}

\author{
U. Warzyńska*, W. Kollek ${ }^{* *}$
}

\begin{abstract}
The paper presents a mathematical model for evaluating pressure pulsation propagation of gas in a pipeline system. The calculations were performed for a natural gas compressor station, in which pressure pulsation was excited by a double-cylinder reciprocating compressor. The mathematical model was based on the acoustic plane wave theory and solved in the frequency domain. The simulation results were compared with experimental test measurements of dynamic pressure changes in the gas installation.
\end{abstract}

\section{Keywords: pressure pulsation, natural gas compressor station}

\section{Introduction}

Pressure pulsation is a dynamic phenomenon in process installations and can have several causes. Most often, pulsation of gas pressure results from the cyclic operation of a positive displacement compressor (i.e. a reciprocating compressor), in particular from instantaneous flow rate. Flow and pressure pulsation causes periodically changing forces to act on a pipeline system. The occurrence of acoustic resonances in a system poses a particular danger to the proper operation of an installation and can lead to a significant increase in excitation amplitudes. Excessive pulsation is an unfavorable phenomenon that can affect mechanical vibrations in the system, leading to fatigue failures, and therefore reducing reliability and increasing the possibility of disasters such as the collapse of the structure or fire due to gas explosion.

According to the well-known API 618 industrial standard, the obligatory method to protect gas installations from excessive pulsation and vibration problem is to perform numerical simulations including all the possible conditions (or at least the worst cases) in which the compressors will operate. The complete methodology for numerical simulations of gas systems is described in the API 618 design approaches and was discussed in (Atkins et al., 2004 and Kollek et al., 2014). The most common methods of modelling pressure pulsation in large pipeline systems are based on the acoustic plane wave theory in time-domain and frequency-domain. The application of the latter method for reciprocating compressors was described by (Cyklis 2001, Soedel 2007 and Zhou et al., 2001). Based on the results of simulations performed as part of the research here presented, it is possible to attenuate the system by introducing orifices or pulsation vessels and dampers.

In the paper, a mathematical model for evaluating pressure pulsation propagation in the pipeline system is presented and compared with on-site experimental test results. The installation chosen for the study comprised a natural gas compressor station. The analyzed system consists of a high-power double-acting two-cylinder reciprocating compressor, pulsation dampers for each side of the cylinders (suction and discharge), a scrubber, a cooler and associated pipelines (Fig. 1).

Urszula Warzyńska, PhD. Eng.: Faculty of Mechanical Engineering, Wrocław University of Science and Technology, Łukasiewicza 5; 50-371 Wrocław; PL, urszula.warzynska@pwr.edu.pl

** Wacław Kollek, Prof.: Faculty of Mechanical Engineering, Wrocław University of Science and Technology, Łukasiewicza 5; 50-371 Wrocław; PL, waclaw.kollek@pwr.edu.pl 


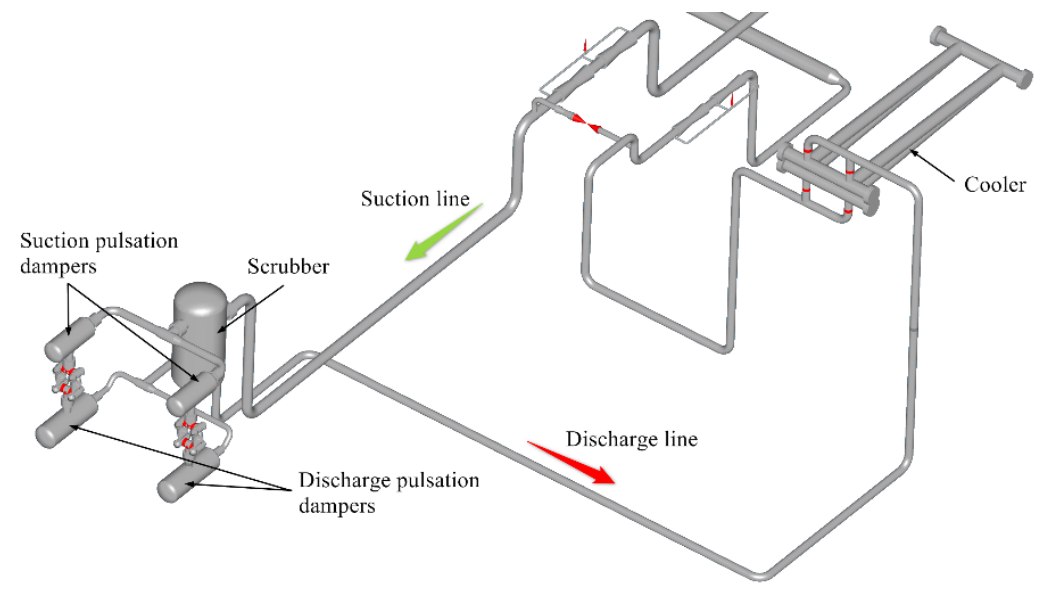

Fig. 1: Geometrical model of the analyzed part of the natural gas compressor station.

\section{Theoretical fundamentals}

The flow of gas in a three-dimensional compressor system generally is described by mass, momentum and energy conservation equations, which include the Navier-Stokes, continuity and state equations. However, due to the size of industrial installations, it is common to reduce the mathematical description to the one-dimensional problem. The geometric spatial model is replaced by pipe sections having specific lengths and cross-sections. The basic condition for reducing the model from the 3D to the 1D description is the assumption that gas parameters are the same at each cross-section of the pipeline. By neglecting gas viscosity and thermal conductivity in the N-S equations, it is possible to obtain the Euler equations. With further assumption that the flow velocity is much lower than the speed of sound, the Euler equations reduce to the wave equation.

The use of the plane wave equation requires the following assumptions:

- constant and variable component of each variable can be separated over time,

- values of variable components are small compared to average (below $\pm 20 \%$ of the average pressure),

- when deriving wave relationships, constant component is omitted and only variable components are modeled in time, assuming the possibility of applying the superposition principle,

- the flow is one-dimensional, and waves of pressure pulsation do not form transverse modes, i.e. no pulsations perpendicular to the axis are generated in a pipeline.

Pressure pulsation can be described by the damped wave equation in the form:

$$
\frac{\partial^{2} \xi}{\partial t^{2}}+\bar{v} \frac{\partial \xi}{\partial t}=c^{2} \frac{\partial \xi}{\partial x^{2}}
$$

where: $\xi$ - displacement in x-axis, $\bar{v}$ - equivalent viscous damping coefficient, $c$ - speed of sound. The value of $\bar{v}$ can be determined from the Helmholtz-Stokes model:

$$
\bar{v}=\frac{2}{D} \sqrt{2 v \omega}
$$

where: D - effective diameter of a pipe, $v$ - kinematic viscosity, $\omega$ - angular frequency.

The wave equation solution is known in the form of:

$$
\xi(x, t)=A_{1} e^{-a x} e^{j(\omega t-k x)}+B_{1} e^{a x} e^{j(\omega t+k x)}
$$

where: $k$-wave number: $k=\frac{\omega}{c}=\frac{2 \pi}{\lambda}, a$ - damping factor: $a=\frac{\bar{v}}{2 c}, j$-imaginary unit: $j=\sqrt{-1}, A_{l}, B_{l}$ - complex constants calculated on the basis of boundary conditions.

Defining the wave propagation coefficient as $\gamma=a+j k$ and taking into account the above relations, the equations of pressure and flow pulsation may be obtained:

$$
\begin{gathered}
p(x, t)=-\rho c^{2} \frac{\partial \xi}{\partial x}=\rho c^{2} \gamma\left[A_{1} e^{-\gamma x}-B_{1} e^{\gamma x}\right] e^{j \omega t} \\
q(x, t)=j \omega S\left[A_{1} e^{-\gamma x}+B_{1} e^{\gamma x}\right] e^{j \omega t}
\end{gathered}
$$




\subsection{Mathematical model of the gas installation}

For the purpose of analytical description of gas pressure pulsation, the discharge section of the compressor system was analyzed. Fig. 2 shows a schematic of the installation described by tabular elements with specified circular cross-sectional area and length. The inlet function of flow pulsation at each of the compressor cylinders (Q01, Q016) was known from the crankshaft mechanism kinematics and $\mathrm{p}-\mathrm{V}$ diagrams. Gas pressure pulsation dampers with internal choking tubes were mounted at the discharge side of the cylinders. Natural gas transported through the two compressor cylinders was directed into the manifold, and then into the cooler (anechoic boundary condition, impedance $\mathrm{Z}=0$ ). The model was written in the Matlab environment.

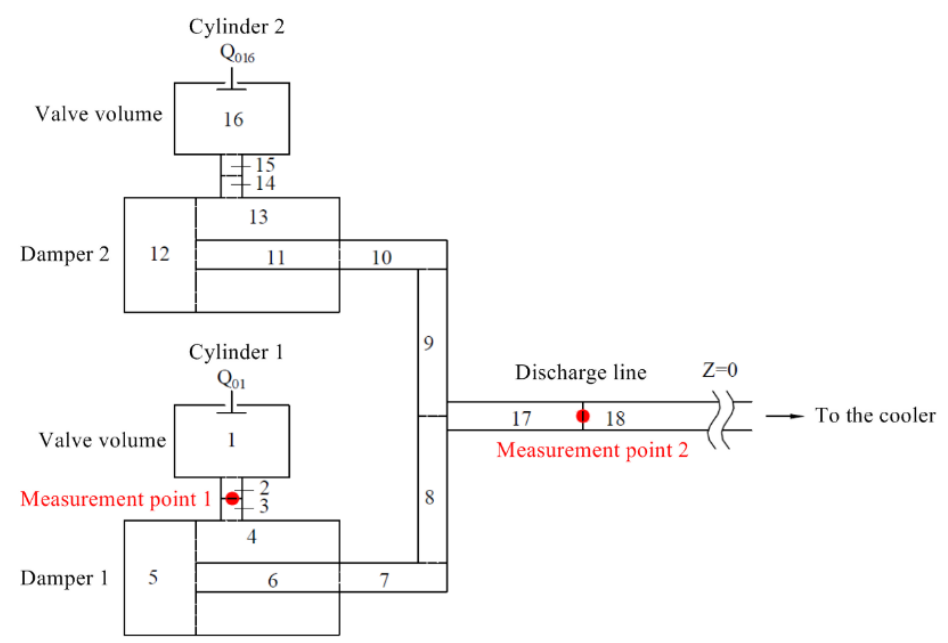

Fig. 2: Schematic of the two cylinder compressor discharge system for the analytical model.

The wave equations of the pulsating volumetric flow rate and pressure were defined by the four-pole transfer matrices integrated into an overall system matrix composed of linear equations with complex coefficients and variables:

$\left[\begin{array}{l}Q_{01} \\ P_{01}\end{array}\right]=M_{1} \cdot M_{2} \cdot M_{3} \cdot F_{4} \cdot F_{5} \cdot M_{6} \cdot M_{7} \cdot M_{8} \cdot T \cdot M_{9} \cdot M_{10} \cdot M_{11} \cdot F_{12} \cdot F_{13} \cdot M_{14} \cdot M_{15} \cdot M_{16} \cdot\left[\begin{array}{l}Q_{L 16} \\ P_{L 16}\end{array}\right](6)$

In the above equation, the symbol $M$ denotes acoustic elements with two inputs and two outputs:

$$
M_{i}=\left[\begin{array}{ll}
A_{i} & B_{i} \\
C_{i} & D_{i}
\end{array}\right], i=1,2 \ldots 18
$$

where: $A_{i}=\cosh \gamma_{i} L_{i}=D_{i} ; B_{i}=\frac{j \omega S_{i}}{\rho_{0} c_{0}^{2} \gamma_{i}} \sinh \gamma_{i} L_{i} ; C_{i}=\frac{\rho_{0} c_{0}^{2} \gamma_{i}}{j \omega S_{i}} \sinh \gamma_{i} L_{i} ; L$ - effective length, $S$ - crosssectional area.

The symbols $F_{4}, F_{5}, F_{12}$ and $F_{13}$ in the equation (6) refer to the chambers of pulsation dampers, i.e. oneside closed elements, in which $\mathrm{Q}_{\mathrm{L}}=0$. The symbol $T$ (tee) denotes the branch of the installation corresponding to the discharge pipeline.

\section{Comparison of the model and test results}

Dynamic pressure changes in the system were measured on-site with the use of dedicated measurement equipment, which meets the stringent requirements of operation in the hazardous conditions. Pressure sensors were mounted in the discharge damper inlet nozzle (measurement point 1, Fig. 2) and in the pipeline section upstream of the cooler (measurement point 2, Fig. 2). The operating conditions were as follows: suction pressure 34 bar, discharge pressure 41 bar, suction temperature $7{ }^{\circ} \mathrm{C}$, discharge temperature $23{ }^{\circ} \mathrm{C}$ and rotational speed $704 \mathrm{rpm}$.

The graphs below (Fig. 3) present the results of pressure pulsation in the form of amplitude-frequency spectra obtained from the analytical model and compared with the values measured during the experimental study in the gas compressor station. The graphs show the peak-to-peak (double amplitude) values of the pressure pulsation versus the frequency of excitation (resulting from the compressor's speed for a given operating point). 

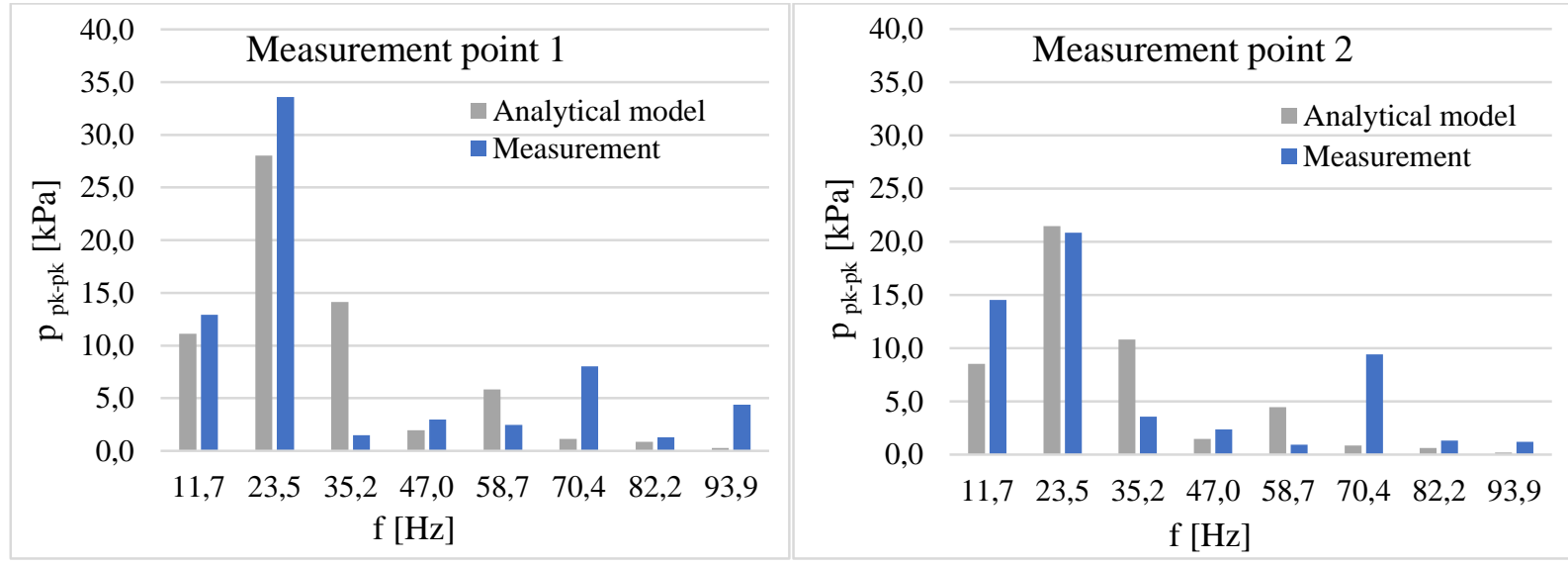

Fig. 3: Comparison of analytical model and measurement of pressure pulsation.

In the investigated case of a two-cylinder double-acting compressor arranged in a balanced-opposed configuration characterized by an adjacent pair of crank throws that are $180^{\circ}$ out of phase, the dominant frequency with the highest amplitude of pulsation is the second harmonic of excitation. According to the API 618 std., it is crucial to damp two first harmonics of the pressure amplitude spectrum to avoid acoustic resonance in a system. For the analyzed operating conditions, the mathematical model shows a good agreement with the measured values in the first two dominant harmonics. The second harmonic of pressure pulsation excited by the compressor piston (at measurement point 1) was damped by the discharge damper (at measurement point 2) by $12.7 \mathrm{kPa}$. The difference between the measured and the simulated values for the dominant amplitude in the pipeline downstream of the damper (at measurement point 2) was less than $3 \%$.

\section{Conclusions}

The article described a mathematical model for pressure pulsation propagation in a gas compressor station. The calculated values of the pressure pulsation amplitudes and frequency were in good agreement with the measured data for the dominant harmonics. The evaluation of the first two harmonic amplitudes was important in the analysis due to the possibility of causing an acoustic resonance in case a coincidence occurs with natural frequencies of the pipeline system. The presented mathematical model will be further developed in order to test different viscous damping coefficients, and also a wider range of operating points will be measured to check the accuracy of the model for different initial conditions. The biggest advantage of the presented method of pressure pulsation modelling is the robustness of calculations for a wide range of compressor operating conditions. Based on the amplitude-frequency spectra, it is possible to properly design pulsation dampers.

\section{References}

API 618 std. (2011) Reciprocating Compressors for petroleum, chemical, and gas industry services, Fifth Edition, American Petroleum Institute, Washington, D.C.

Atkins K. E., Pyle A. S. and Tison J. D. (2004) Understanding the pulsation \& vibration control concepts in the new API 618 fifth edition. Gas Machinery Conference in Albuquerque, New Mexico.

Cyklis, P. (2001) Experimental identification of the transmittance matrix for any element of the pulsating gas manifold. Journal of Sound and Vibration, 244, 5, pp. 859-870.

Kollek W., Harnatkiewicz P., Lubecki Sz., Osiński P. and Radziwanowska U. (2014) Methods of pressure pulsation and vibration analysis in industrial network for gas transmission. Inżynieria Maszyn, R. 19, z. 2 (in Polish).

Soedel W. (2007) Sound and vibrations of positive displacement compressors. CRC Press.

Zhou W., Kim J. and Soedel W. (2001) New iterative scheme in computer simulation of positive displacement compressors considering the effect of gas pulsations. Transactions of the ASME, Vol. 123, pp. 282-288. 